\section{Implementación de la vacunación contra el virus papiloma humano en Chile: una mirada desde los determinantes sociales de la salud "ingreso"y "género"}

\author{
LORETO FERNÁNDEZ GONZÁLEZ $Z^{1,2, a}$
}

\section{HPV vaccine implementation in Chile: an appraisal from the social determinants of health model}

Cervical cancer is the fourth most common neoplasm in women worldwide and its incidence is associated with profound social inequities. In Chile, it is the second cause of death in women of reproductive age. The Chilean clinical guideline identifies the vaccine against Human Papillomavirus (HPV) as the main preventive measure. Since 2014, the Ministry of Health has implemented free immunization against HPV for girls and female adolescents. This article critically analyzes this public policy from the viewpoint of health equity, using as framework the Social Determinants of Health Model. Specifically, we address the structural determinants of income and gender, which act as material and social barriers for achieving immunization, affecting protection against cervical cancer. These barriers correspond to the high cost of the vaccine, and social attitudes/cultural beliefs towards sexual behavior in Latin America and Chile that affect the acceptability of vaccination. The Social Determinants of Health Model constitutes a useful tool for identifying health inequities and understanding public policy from an equity viewpoint that complements the biomedical and epidemiological understanding of disease. In this topic, the initiative aims to strengthen the idea of health as a human right and health promotion as an essential function of public health policy.

(Rev Med Chile 2017; 145: 1605-1609)

Key words: Health Equity; Health Policy; Papillomavirus Vaccines; Social Determinants of Health; Uterine Cervical Neoplasms.

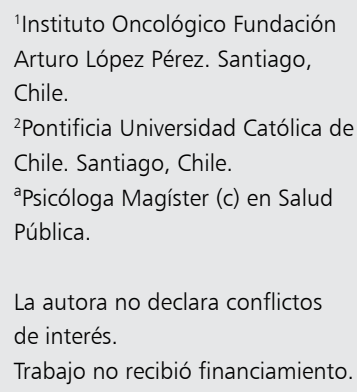

Recibido el 14 de julio de 2017 , aceptado el 15 de diciembre de 2017.

Correspondencia a:

Loreto Fernández

Ricardo Lyon 249, depto. 31,

Santiago, Chile.

loretofernandez2@gmail.com
E 1 cáncer de cuello uterino $(\mathrm{CaCu})$ es el cuarto cáncer más común en mujeres a nivel mundial, con $87 \%$ de las muertes producidas por este en regiones menos desarrolladas, lo que da cuenta de su magnitud como problema de salud pública y la inequidad que implica ${ }^{1}$. En Chile, es la segunda causa de muerte por cáncer en mujeres en edad reproductiva, produciendo alrededor de 600 muertes anuales, de acuerdo a datos del Ministe- rio de Salud (MINSAL) ${ }^{2}$. El CaCu es la patología considerada en las Garantías Explícitas en Salud (GES) $\mathrm{N}^{\circ} 3$, es decir, su diagnóstico y tratamiento es una garantía que constituye un derecho en salud para las personas en Chile. Dentro de la guía clínica correspondiente ${ }^{3}$, podemos identificar como la principal medida preventiva primaria, la vacunación contra el virus papiloma humano (VPH), el cual constituye un factor necesario para 
el desarrollo del CaCu. A partir del año 2014, el MINSAL implementó la vacunación contra el VPH como política sanitaria, con el objetivo de disminuir el riesgo de desarrollar $\mathrm{CaCu}$. Si bien la vacuna estaba disponible en Chile desde 2013, su alto costo constituía una barrera importante para su administración. En ese sentido, la medida del MINSAL "como parte de una política pública del Estado, garantiza en Chile que todas las niñas puedan acceder a ella en forma gratuita, dando un nuevo paso en equidad en Salud"2. De acuerdo a datos del MINSAL, el costo estimado de la vacuna para el Estado de Chile en 2014 sería de $\$ 1.200$ millones de pesos, y el alcance de niñas vacunadas se estimó en 120.000 para ese mismo año, y a partir de 2015, una administración de 250.000 dosis anuales ${ }^{4}$.

El propósito de este artículo es analizar esta política sanitaria desde el Modelo de los Determinantes Sociales en Salud (DSS), en particular, desde los determinantes estructurales de Ingreso y Género.

\section{Determinantes sociales en salud en la vacunación contra el VPH}

Los DSS se definen como las circunstancias en que las personas nacen, crecen, viven, trabajan y envejecen, incluido el sistema de salud ${ }^{5}$. Los DSS explican la mayor parte de las inequidades sanitarias, es decir, aquellas desigualdades consideradas injustas y evitables de un país, y entre países ${ }^{6}$. Estos DSS pueden ser estructurales o intermedios, según la función que ocupan en las condiciones económicas, políticas, materiales y psicosociales que moldean la salud de los individuos. De acuerdo a Hirmas $^{7}$, las barreras u obstáculos para lograr la atención sanitaria más reportados en la literatura son las correspondientes a aceptabilidad y accesibilidad, seguidas de las barreras de contacto con el servicio de salud, y disponibilidad. En el caso de la implementación de la vacunación contra el VPH en Chile, los DSS respectivos constituyen el Ingreso (accesibilidad) y Género (aceptabilidad). Ambos son DSS estructurales en tanto generan y fortalecen la estratificación social, dificultando la inmunización que pretende subsanar la política del MINSAL y, por ende, a la protección contra el $\mathrm{CaCu}$. Estas barreras pueden traducirse brevemente en: alto costo de la vacuna, y actitudes/ conductas sexuales asociadas al género en América Latina y Chile.

\section{Ingreso}

En Chile, de acuerdo a datos del INE del año 2015, el promedio mensual de ingresos entre los ocupados era de $\$ 505.477$, con un ingreso mensual mediano de $\$ 340.000$ pesos, y con una brecha entre mujeres y hombres cercana a $30 \%$ en desmedro de las mujeres ${ }^{8}$. A esto debemos agregar que Chile posee un elevado gasto de bolsillo en salud, cercano a $40 \%{ }^{9,10}$. Por su parte, el costo de la vacuna contra el VPH ronda los $\$ 80.000$ pesos cada dosis, requiriéndose dos dosis en un lapso de 24 meses para lograr la inmunización ${ }^{11}$. De este modo, su precio resultaba prohibitivo para un porcentaje significativo de chilenos, y en especial de chilenas, al ser sus salarios casi un tercio más bajos. Considerando que las destinatarias de la vacuna, además, son menores de edad en etapa escolar, es decir, sujetos que no reciben ingresos, la vacuna se consideraba como un bien de alto costo, no esencial, fuera del alcance de la mayoría de las familias. El incluir la vacuna en la guía clínica del GES CaCu, constituye una política de salud pública orientada a la prevención primaria, que garantiza el acceso y la protección financiera, sin dejar de lado la oportunidad y la calidad, las otras dos garantías del GES. Sitúa la posibilidad de prevenir el $\mathrm{CaCu}$ como un derecho sanitario para las mujeres, mitigando la inequidad producida por la desigualdad en los ingresos, el gasto de bolsillo en salud, y su impacto sanitario en el caso de este cáncer en particular.

\section{Género}

Es importante hacer la salvedad que no nos referiremos al riesgo asociado al sexo femenino de contraer el VPH, ya que ambos sexos están expuestos a contraer el virus. En este sentido, haremos la distinción entre el riesgo de contraer $\mathrm{VPH}$ y riesgo de desarrollar $\mathrm{CaCu}$, y cómo las inequidades en el campo del género hacen más vulnerables a las mujeres a padecer este cáncer. Es decir, independiente de las razones biológicas que exponen a las mujeres a desarrollar $\mathrm{CaCu}$, hay condiciones sociales o DSS que incrementan la vulnerabilidad de este grupo de la población. Para clarificar este punto es útil acudir a la terminología de Krieger "expresión biológica del género", entendiendo género como el "concepto social de 
convenciones, papeles y comportamientos de carácter cultural asignados a las mujeres y hombres y a los niños y niñas, así como a las relaciones entre ellos." ${ }^{12}$. Esta noción de género, según la autora, incorpora en su comprensión la categoría de poder y cómo este media las relaciones sociales, generando injusticia e inequidad en salud, a través de conductas sexistas ${ }^{12}$.

En el caso de América Latina, el sexismo en desmedro de la participación social y económica, y su impacto en salud, es un fenómeno bien descrito $^{13}$. Como ejemplo tenemos el dato revisado anteriormente en cuanto a la brecha salarial entre hombres y mujeres y el inevitable impacto que esto tiene en el poder adquisitivo y la calidad de vida de las personas. En el campo de la salud sexual/reproductiva y enfermedades transmisibles, la investigación epidemiológica ha prestado más interés al proceso de enfermedad mismo que a las raíces socioculturales en las cuales surge y se propaga. Esto ha ido en desmedro de generar conciencia de las conductas sexuales y la importancia del género y las prácticas sexistas. De acuerdo a Hartigan ${ }^{13}$, en el caso del VPH, factores relacionados al género que juegan en contra de su control epidemiológico son el rechazo masculino al uso del condón, las barreras de acceso a educación sexual en niños y niñas y el valor atribuido a la promiscuidad versus la virginidad según el género.

En esta línea, uno de los mayores debates en torno al VPH ha sido, justamente, la creencia que la vacunación fomentaría la promiscuidad y las relaciones sexuales desprotegidas de las mujeres. De este modo, una importante barrera en la aceptabilidad de la vacuna ha sido el rechazo de ciertos grupos (incluyendo padres de menores en edad de vacunarse) a la idea de que las mujeres pueden tener múltiples compañeros sexuales debido a una baja percepción de riesgo de esta conducta, responsabilizando a la mujer del contagio ${ }^{14,15}$. A pesar de que estudios muestran que la vacunación no aumenta la promiscuidad ${ }^{16}$, sigue siendo un tema controvertido, que en nuestro propio país se ha sentido con fuerza y que deja entrever que el propio $\mathrm{CaCu}$ está ligado al estigma de la promiscuidad femenina ${ }^{17}$. La obligatoriedad de la vacunación por parte del MINSAL, ha conllevado también una campaña educativa que desmitifica que la inoculación incentiva promiscuidad en las mujeres, o que el evitar múltiples compañeros sexuales garantice protección contra el virus. De este modo, el Estado apela al bien común como un fin más relevante que las creencias individuales sobre el virus y la conducta sexual propia ${ }^{11}$.

\section{Discusión}

El estado actual en la región en cuanto a la inmunización contra el VPH da cuenta de avances y desafíos aún pendientes. De acuerdo a lo revisado, la disponibilidad de la vacuna debe, además, sustentarse en la accesibilidad y la aceptabilidad para poder aspirar a una cobertura universal. A la fecha, los países que cuentan con programas de vacunación contra el VPH en las Américas son Argentina, Antigua, Barbados, Belize, Brasil, Bermuda, Chile, Islas Caimanes, Colombia, Ecuador, Guyana, Honduras, México, Panamá, Paraguay, Perú, Puerto Rico, Saba, St. Maarten, Suriname, Trinidad y Tobago y Uruguay. Otros países, tales como Venezuela, aunque tiene incluida la vacuna en su programa nacional, no cuenta con la disponibilidad actualmente ${ }^{18}$. Si bien, luego de que Brasil introdujera la vacuna obligatoria en 2014, la Organización Panamericana de la Salud afirmó que más de $80 \%$ de las niñas americanas tendrían acceso a la vacuna, esta afirmación se basa en el porcentaje de niñas viviendo en los países con programas de vacunación, y no en quienes efectivamente reciben la vacuna. Los datos muestran que la vacuna ha tenido una cobertura preocupantemente baja, con una adherencia menor a la esperada, sobre todo en las dosis posteriores a la primera, según el esquema utilizado. El caso más alarmante es el de Colombia, que obtuvo 97,5\% de inoculación de la primera dosis y $20,4 \%$ en la segunda, disminución que aparentemente responde al reporte de casos con efectos adversos en el país ${ }^{18}$. De este modo se observa que el éxito de la inmunización no sólo responde a la efectividad de la vacuna misma, y tampoco al hecho de recibirla de forma gratuita, sino que la información y la educación para la salud deben constituir un paso esencial para mejorar su aceptabilidad, disminuir las creencias erróneas, y mitigar las prácticas sexistas asociadas a la salud de la mujer en la región. En esta línea, el Consenso para la Prevención del Cáncer Cérvicouterino sitúa a la inmunización y la educación para la salud como las recomendaciones principales en el ámbito de la prevención primaria ${ }^{19}$. 


\section{Conclusión}

La política sanitaria de la obligatoriedad de la vacunación contra el VPH en niñas constituye un esfuerzo del Estado por prevenir el $\mathrm{CaCu}$ y disminuir su incidencia en el futuro. El modelo de los DSS constituye una herramienta útil para identificar inequidades y barreras en salud, y comprender las políticas públicas desde un enfoque de equidad que complementa la mirada biomédica y epidemiológica. En el caso expuesto, la iniciativa busca fortalecer la noción de la salud como derecho y la promoción como una función esencial de la salud pública por parte del Estado. Si bien ciertas limitaciones pueden encontrarse -tales como el riesgo de contraer otras enfermedades asociadas a VPH que afectan a ambos sexos, o la dificultad para vacunar niñas fuera del sistema educativo-, el inmunizar a las mujeres es un paso importante en fomentar la equidad de género y su expresión biológica, y garantizar su acceso a toda la población, con miras a subsanar la jerarquía social y discriminación subyacente a las diferencias de ingreso y prácticas sexistas en la salud sexual femenina. Es imprescindible implementar la vacunación en conjunto con estrategias de educación para la población, incluyendo a los servicios de salud, con miras a lograr una real cobertura universal basada en la aceptabilidad y el conocimiento sobre sus beneficios y reales riesgos.

\section{Referencias}

1. Bychkovsky BL, Ferreyra ME, Strasser-Weippl K, Herold CI, de Lima Lopes G Jr, Dizon DS, et al. Cervical cancer control in Latin America: A call to action. Cancer 2016; 122: $502-14$

2. Ministerio de Salud. Vacunación contra el Virus del Papiloma Humano. 2016 [online] Disponible en: http:// web.minsal.cl/vacunacion-contra-el-virus-del-papiloma-humano/ [Consultado el 30 de abril 2017].

3. Ministerio de Salud [online] Guía Clínica. Cáncer Cervicouterino (CaCu). 2015 Disponible en: http://www. bibliotecaminsal.cl/wp/wp-content/uploads/2016/04/ GPC-CaCU-Final.PLdocx.pdf [Consultado el 30 de abril 2017].

4. Ministerio de Salud. Vacunación contra el Virus del Papiloma Humano. s/f [online] Disponible en: http:// vacunas.minsal.cl/informacion-a-la-comunidad/vacunacion-contra-vph/vacunacion-contra-virus-papiloma-humano-2014/ [Consultado el 30 de abril 2017].
5. Commission of the Social Determinants of Health. Closing the gap in a generation: health equity through action on the social determinants of health. Final Report of the Commission on Social Determinants of Health. Geneva, World Health Organization; 2008.

6. World Health Organization. Determinantes sociales de la salud. 2017 [online]. Disponible en: http://www.who. int/social_determinants/es/ [Consultado el 30 de abril 2017].

7. Hirmas Adauy M, Poffald Angulo L, Jasmen Sepúlveda AM, Aguilera Sanhueza X, Delgado Becerra I, Vega Morales J. Barreras y facilitadores de acceso a la atención de salud: una revisión sistemática cualitativa. Rev Panam Salud Pública 2013; 33(3): 223-9.

8. Instituto Nacional de Estadísticas (s.f.) Encuesta Suplementaria de Ingresos [online]. Disponible en: http:// www.ine.cl/estadisticas/ingresos-y-gastos/esi [Consultado el 28 de octubre de 2017].

9. Cid Pedraza C, Prieto Toledo L. El gasto de bolsillo en salud: el caso de Chile, 1997 y 2007. Rev Panam Salud Publica 2012; 31 (4): 310-6.

10. Cid Pedraza C. Financiamiento agregado de la salud en Chile: año 2008. Cuad Méd Soc (Chile) 2011; 51 (3): 143-50.

11. Gobierno de Chile (2014). Las claves sobre la vacunación contra el Virus Papiloma Humano [online]. Disponible en: http://www.gob.cl/juntos-por-ellas-todo-sobre-la-vacunacion-contra-el-virus-papiloma-humano/ [Consultado el 30 de abril 2017].

12. Krieger N. Glosario de epidemiología social. Revista Panamericana de Salud Pública 2002; 11 (5-6): 480-90. https://dx.doi.org/10.1590/S1020-49892002000500028.

13. Hartigan P. Enfermedades transmisibles, género, y equidad en la Salud. Organización Panamericana de la Salud. Harvard Center for Population and Development Studies.; 2000. ISBN 927532287 2. Disponible en: http:// iris.paho.org/xmlui/handle/123456789/807 [Consultado el 30 de abril 2017].

14. Wiesner C, Piñeros M, Trujillo LM, Cortés C, Ardila J. Human papillomavirus (HPV) vaccine acceptability amongst parents of adolescents in four Colombian areas. Revista de Salud Pública 2010; 12 (6): 961-73.

15. Verdugo MKG, Nacach AZ, Campos MCA. Aceptación de la vacuna contra el virus del papiloma humano por parte de madres de hijas entre 9 y 13 años de edad. Ginecol Obstet Mex 2013; 81: 645-51.

16. Bednarczyk R. Human Papillomavirus Vaccine and Sexual Activity. JAMA Internal Medicine 2015; 175 (4): 624. Disponible en: https://jamanetwork.com/journals/ jamainternalmedicine/article-abstract/2109851?redirec$\mathrm{t}=$ true. [consultado el 28 de octubre de 2017]. 
17. Shepherd MA, Gerend MA. The blame game: cervical cancer, knowledge of its link to human papillomavirus and stigma. Psychology \& health 2015; 29 (1): 94-109.

18. Nogueira-Rodrigues A, Bukowski A, Paulino E, St Louis J, Barrichello A, Sternberg C, Goss PE. An alert to Latin America: Current human papillomavirus vaccination trends highlight key barriers to successful implementation. Cancer 2017; 123: 2193-9.

19. Carrillo FJO, de Regil DBG, Jiménez MTV. Infección por virus del papiloma humano en mujeres y su prevención. Gaceta Mexicana de Oncología 2015; 14 (3): 157-63. 